\title{
Diagnóstico diferencial das insuficiências respiratórias agudas
}

\section{Acute respiratory disorders in the neonatal period}

\author{
Lilian dos S. Rodrigues Sadeck(1)
}

\begin{abstract}
Sadeck LSR. Diagnóstico diferencial das insuficiências respiratórias agudas. Rev Med (São Paulo) 2003 jan.-dez.;82(1-4):40-5.

RESUMO: Os recém-nascidos com insuficiência respiratória aguda são responsáveis pelo maior número de internações em unidades de terapia intensiva neonatal, sendo inclusive a principal causa de óbito, especialmente naqueles que ocorrem nos primeiros dias de vida. Além disso, estes RN podem evoluir com seqüelas, como a doença pulmonar crônica e a displasia broncopulmonar, que poderão comprometer a qualidade de vida futura. Esta revisão aborda os dados mais relevantes para se realizar o diagnóstico diferencial das insuficiências respiratórias agudas no período neonatal, levando em conta os dados epidemiológicos, quadro clínico, radiológico e evolutivo.
\end{abstract}

DESCRITORES: Insuficiência respiratória/diagnóstico. Insuficiência respiratória/complicações. Insuficiência respiratória/etiologia. Doenças respiratórias/diagnóstico. Diagnóstico diferencial. Recémnascido.

\section{INTRODUÇÃO}

A insuficiência respiratória no período neonatal pode ser uma manifestação clínica decorrente de diferentes causas, não necessariamente pulmonar. As situações encontradas mais freqüentemente estão enumeradas no Quadro $1^{1}$.

Os sinais clínicos caracterizam-se por: taquipnéia (frequiência respiratória $>60$ movimentos por minuto), hiperpnéia, bradipnéia, tiragem intercostal, subcostal, retração esternal, gemência, batimento de asa de nariz, hipo ou atonia, cianose ou palidez. Estes sinais podem aparecer isolados ou em várias combinações.
Nos casos de insuficiência respiratória por problemas primariamente cardíacos, os sinais de desconforto respiratório são, geralmente, caracterizados por uma taquipnëia sem outros sinais de esforço respiratório e estão associados a outras manifestações, como hepatomegalia, taquicardia, ritmo de galope, máperfusão periférica, ausência de pulsos femorais, alterações de pressão arterial, sopros cardíacos, alterações das bulhas e cianose central. Dentre as cardiopatias congênitas que apresentam manifestações precocemente e podem entrar como diagnóstico diferencial de desconforto respiratório,

\footnotetext{
(1) Médica Encarregada do Setor de Alto Risco do Berçário Anexo à Maternidade do Hospital das Clínicas da FMUSP. Doutora em Pediatria pela FMUSP.

Endereço para correspondência: R. Dr. Augusto de Miranda, 1092/83. CEP: 05026-001 - São Paulo, SP. e-mail: liliansadeck@ sti.com.br
} 
destacam-se: transposição dos grandes vasos da base, síndrome da hipoplasia do coração esquerdo, coartação da aorta complexa, persistência do canal arterial e defeitos septais. Além da suspeita clínica o diagnóstico da cardiopatia pode ser feito através da radiografia, eletrocardiograma, ecocardiograma e ecodopplerfluxometria ${ }^{2}$.

Quadro 1. Principais causas de desconforto respiratório agudo no período neonatal

\section{Problemas pulmonares}
a) Doença de membranas hialinas (DMH)
b) Taquipnéia transitória do recém-nascido (TTRN)
c) Síndrome de pulmão úmido
d) Síndrome de aspiração meconial (SAM)
e) Outras síndromes aspirativas
f) Pneumonias
g) Pneumotórax, enfisema intersticial, pneumomediastino
h) Atelectasia
i) Seqüestro pulmonar
j) Hipoplasia pulmonar, hérnia diafragmática
k) Síndromes malformativas (enfisema lobar congênito, doença adenomatosa cística)
1) Derrames, quilotórax
m) Hemorragia pulmonar

\section{Problemas cardíacos}
a) Cardiopatia congênita
b) Hipertensão pulmonar persistente
c) Isquemia miocárdica pós-asfíxica
d) Hidropisia fetal secundária à isoimunização
e) Hidropisia não imune
f) malformações arteriovenosas
g) transfusão feto-fetal

\section{Problemas não cardio-respiratório}
a) Metabólicos (hipoglicemia, hipocalcemia, acidose metabólica)
b) Hipotermia, hipertermia
c) Sepse
d) Leucinoses
e) Hiponatremia, hipernatremia
f) Condrodistrofias

\section{Problemas neurológicos}
a) Asfixia perinatal
b) Apnéia do pré-termo
c) Depressão por drogas
d) Hemorragia intra-craniana
e) Meningite
f) Doença de Werdnig-Hoffman
g) Encefalocele

\section{Problemas do trato respiratório alto}
a) Atresia de coanas, edema nasal
b) Macroglossia, micrognatia; síndrome de Pierre Robin
c) Bócio congênito, higroma cístico
d) Membrana laríngea, estenose subglótica, hemangioma, laringomalácia
e) Paralisia de cordas vocais
f) Traqueomalácia, fístula tráqueo-esofágica
g) Estenose traqueal, estenose brônquica 
A síndrome de hipertensão pulmonar persistente ${ }^{3}$ (SHPP) é uma síndrome clínica caracterizada por pressão elevada em artéria pulmonar, "shunt" direita-esquerda através do forame oval e/ou canal arterial e presença de cianose central grave, com labilidade de oxigenação. O quadro clínico mais característico é de uma insuficiência respiratória progressiva nas primeiras horas de vida, em crianças de termo ou pós-termo, acompanhada de cianose mantida ou de aparecimento durante os esforços (choro, manipulação) e a presença de antecendentes perinatais de: asfixia grave por sofrimento fetal agudo ou crônico, sepse, hérnia diafragmática, hipoplasia pulmonar ou lesões torácicas congênitas. Geralmente, os sinais clínicos de desconforto respiratório são desproporcionais à intensidade da cianose. Pode apresentar sinais de insuficiência cardíaca, choque ou asfixia grave. O diagnóstico pode ser feito através do achado de um diferencial de pressão arterial de oxigênio $\left(\mathrm{PaO}_{2}\right)$ superior a 10 a $15 \mathrm{mmHg}$, entre uma amostra gasométrica colhida de artéria pré-ductal (artéria radial direita ou temporal) e outra de artéria pós-ductal (artéria umbilical, femoral ou tibial), simultaneamente. Quando não se constata esta diferença entre os dois exames, a SHPP não pode ser afastada, pois o "shunt" poderá estar ocorrendo preferencialmente pelo forame oval. Nestes casos o diagnóstico apenas poderá ser feito através de ecocardiografia bidimensional e doppler, no qual observase um "shunt" direita-esquerda através do forame oval ou canal arterial e pressão de artéria pulmonar mais elevada do que a da aorta. Quando esta não for disponível, pode-se realizar uma prova terapêutica, que consiste em: hiperoxiahiperventilação $\left(\mathrm{FiO}_{2}\right.$ de $100 \%$ com FR acima de $\left.80 \mathrm{mpm}\right)$. O objetivo deste teste é diminuir a pressão arterial de $\mathrm{CO}$ $\left(\mathrm{PaCO}_{2}\right)$ até atingir um nível crítico $(15 \text { a } 20 \mathrm{mmHg})^{2}$, produzindo uma alcalose respiratória que, por sua vez, leva à vasodilatação pulmonar e melhora da oxigenação.

Nos casos de insuficiência respiratória, deve-se sempre investigar a presença de distúrbios metabólicos, pois estes podem ser tanto causa do distúrbio, como também um fator associado, que agrava o quadro de base ${ }^{2}$.

Em problemas neurológicos ${ }^{2}$, geralmente os sinais respiratórios estão associados aos decorrentes de comprometimento do sistema nervoso, tais como: diminuição da atividade espontânea, apnéia, hipotonia, hiporreflexia, irritabilidade, convulsões, nistagmo, queda de hematócrito (nos casos de hemorragia intracraniana), abaulamento de fontanela. O diagnóstico poderá ser feito através do exame do LCR, ultra-sonografia de crânio e tomografia computadorizada de crânio.

Afastadas as outras causas de desconforto respiratório por problemas extra-pulmonares, ainda resta a dificuldade clínica de diferenciar a patologia pulmonar responsável pela sintomatologia.

Cada caso clínico deverá ser avaliado globalmente, iniciando-se pelos antecedentes maternos, condições e tipo de parto, características do RN, época do aparecimento do desconforto, evolução, exame clínico, radiológico e laboratorial. A seguir será exposto cada um destes itens:
1. Antecedentes maternos: os dados maternos podem muitas vezes sugerir a causa do desconforto ${ }^{4}$ A prematuridade em gestação anterior ou nesta (idade gestacional < 34 semanas de gestação) e a presença de diabetes mellitus estão associados a uma maior incidência de doença de membranas hialinas (DMH). A presença de bolsa rota há mais de 18 horas antes do parto, associada a sinais de infecção materna, tais como: corioamnionite, febre, fisometria, ou com evidência de bactérias no líquido amniótico, pode estar relacionada a uma pneumonia por Streptococcus do grupo B ou por bactérias Gram negativas no RN. Antecedentes de infecção materna do trato urinário também devem ser valorizados quando suspeita-se de um quadro de infecção como responsável pela insuficiência respiratória. $\mathrm{O}$ uso de medicamentos pela mãe durante a gestação, tais como: os iodados, os antiinflamatórios (AAS, indometacina) e reserpina, podem causar bócio congênito, SHPP e obstrução nasal, respectivamente. A informação referente à quantidade de líquido amniótico também é importante. O polihidrâmio pode estar associado a malformações do trato gastrointestinal do tipo atresia de esôfago ou fístulas tráqueo-esofágicas e o oligoâmnio, à hipoplasia pulmonar;

2. Condições e tipo de parto: estes dados podem sugerir ou afastar as possíveis hipóteses diagnósticas. $\mathrm{O}$ desencadeamento do trabalho de parto acelera a reabsorção do líquido intra-alveolar ${ }^{5}$, através de liberação de fatores humorais, portanto, nos casos em que este não ocorrer aumenta a chance de patologias pulmonares decorrentes da demora de reabsorção deste líquido, isto é, taquipnéia transitória do RN e síndrome de pulmão úmido. O parto cesáreo geralmente está associado à maioria dos desconfortos respiratórios, exceto nos casos de pneumonias. A asfixia perinatal grave está associada a DMH, síndrome de pulmão úmido, SHPP ou síndrome de aspiração meconial (SAM), dependendo da idade gestacional do RN. O uso de analgésicos durante o final do trabalho de parto parece aumentar a incidência de taquipnéia transitória do RN. A anestesia geral ou derivados de morfina na hora do parto podem causar depressão respiratória e asfixia perinatal. A reanimação mal conduzida pode causar asfixia perinatal, pneumotórax, enfisema intersticial, pneumomediastino;

3. Características do $R \boldsymbol{N}$ : a idade gestacional e a adequação de peso para esta idade são muito importantes para colaborar na formulação de uma hipótese diagnóstica $^{6}$. A prematuridade, especialmente nos casos de RN com idade gestacional menor do que 34 semanas, sugere DMH ou síndrome de pulmão úmido ${ }^{7}$. Os RN com idade gestacional limítrofe ao termo, entre 35 a 36 semanas, têm maior risco de desenvolver um quadro de taquipnéia transitória do $\mathrm{RN}$, enquanto o grupo de $\mathrm{RN}$ pós-termo têm maior risco de desenvolver síndromes aspirativas, especialmente síndrome de aspiração meconial, ou síndrome de hipertensão pulmonar 
persistente. O RN pré-termo (RNPT) adequado para a idade gestacional tem maior risco de desenvolver doença de membranas hialinas do que o RNPT pequeno para a idade gestacional ${ }^{7}$. Este último, quando apresenta insuficiência respiratória, esta mais frequentemente é decorrente de pletora neonatal, problemas infecciosos, pneumonias aspirativas ou malformações congênitas. A presença de outros sinais clínicos de alterações cromossômicas, displasias esqueléticas, obstrução de vias aéreas superiores, também podem nos ajudar a fazer o diagnóstico da causa da insuficiência respiratória;

\section{4. Época de aparecimento dos sinais de} insuficiência respiratória, sinais clínicos e sua evolução: a maioria destas patologias apresentam sinais clínicos de insuficiência respiratória logo após o nascimento, não sendo de muita ajuda para diferenciá-los (Quadro 2$)^{2}$. Devemos ressaltar apenas que, na $\mathrm{DMH}^{7}$, apesar da maioria dos casos desenvolver sinais clínicos logo após o nascimento, alguns podem vir a apresentá-los num período de até duas horas. Os casos de pneumonia precoce podem vir a evidenciar manifestações clínicas até o terceiro dia de vida. Muitas vezes o que mais ajuda para fechar o diagnóstico da insuficiência respiratória é a evolução clínica nos primeiros dias. Nos casos de taquipnéia transitória do $\mathrm{RN}^{8}$ os sinais clínicos já estão presentes desde o nascimento, pois são decorrentes de uma demora na reabsorção do líquido intra-alveolar. Inicialmente, apresentam taquipnéia, com frequência respiratória de 80 a $100 \mathrm{mpm}$, tiragem intercostal, retração esternal, cianose e necessidade de oxigenoterapia. Nas primeiras 24 horas de vida evoluem com melhora do quadro clínico, mantendo apenas a taquipnéia por até 48 horas. Nos casos de síndrome de pulmão úmido ${ }^{6}$ os sinais de insuficiência respiratória também aparecem logo após o nascimento, e também são decorrentes de uma demora da reabsorção do líquido intra-alveolar, nestes casos mais intensa, pois ocorre em RNPT. O sinal sempre presente nestes casos é a taquipnéia associado a gemência, tiragem intercostal e batimento de asa de nariz. Muitas vezes o quadro clínico e radiológico desta patologia confunde-se com o da doença de membranas hialinas e o que ajuda a diferenciar as duas patologias é a evolução clínica, pois os casos com líquido intra-alveolar evoluem com melhora dos sintomas em 24 horas, ao contrário da $\mathrm{DMH}$, que se intensifica nesse período. Na DMH a criança também pode apresentar sinais de comprometimento respiratório desde o nascimento, caracterizado por gemência, batimento de asa de nariz, tiragem intercostal, retração esternal, balancim tóracoabdominal. Estes parâmetros são utilizados para avaliar a evolução clínica do desconforto respiratório através do boletim de Silverman-Anderson (Quadro 3). Esta patologia é causada pelo aparecimento progressivo de microatelectasias decorrente da falta de produção de surfactante pelos pneumatócitos tipo II imaturos ${ }^{7}$. Sua evolução natural é caracterizada por uma piora clínica e radiológica nas primeiras 24 a 48 horas após o parto, seguida de uma estabilização do quadro até 72 horas, para então começar a melhorar. Ë uma patologia do pré-termo com menos de 34 semanas de idade gestacional, associado a uma asfixia perinatal grave, raça branca, sexo masculino e filho de mãe com diabetes gestacional ou insulino dependente. Outros fatores, como a imaturidade morfológica do pulmão, pneumotórax, persistência do canal arterial, infecção, síndrome de hipertensão pulmonar persistente e apnéia, podem prolongar a insuficiência respiratória;

Quadro 2. Idade de aparecimento da insuficiência respiratório relacionado com o diagnóstico (Adaptado de Bhutani, VK, 1996) ${ }^{2}$

\begin{tabular}{|c|c|c|}
\hline & Aparecimento agudo & Aparecimento insidioso \\
\hline \multirow[t]{12}{*}{ Aparecimento desde a sala de parto } & Apnéia & Resolve em 12 horas \\
\hline & Obstrução de vias aéreas & Hipotermia \\
\hline & Malformação congênita & Desconforto respiratório leve \\
\hline & Hipoplasia pulmonar & Melhora em 24-48 horas \\
\hline & Derrame pleural, hidropisia & S. Pulmão úmido \\
\hline & Massa intratorácica & Taquipnéia transitória \\
\hline & & Piora em 12 horas \\
\hline & & $\mathrm{DMH}$ \\
\hline & & SAM \\
\hline & & SHPP \\
\hline & & Pneumonia \\
\hline & & Cardiopatia congênita \\
\hline \multirow[t]{5}{*}{ Aparecimento na primeira semana } & Ar fora do pulmão & Pneumonia nosocomial \\
\hline & Apnéia da prematuridade & Cardiopatia congênita \\
\hline & Apnéia secundária & PCA \\
\hline & Hemorragia pulmonar & Malformação intrapulmonar \\
\hline & Sindrome aspirativa & Distensão abdominal \\
\hline
\end{tabular}


Rev Med (São Paulo) 2003 jan.-dez.;82(1-4):40-5.

Quadro 3. Boletim de Silverman-Anderson

\begin{tabular}{llll}
\hline Parâmetros & $\mathbf{0}$ & $\mathbf{1}$ & $\mathbf{2}$ \\
\hline Gemência & ausente & audível com esteto & audível sem esteto \\
Batim. de asa nariz & ausente & discreto & acentuado \\
Tiragem intercost. & ausente & 3 últimos intercost. & mais de 3 intercost. \\
Retração esternal & ausente & discreta & acentuada \\
Balancim & ausente & discreto & acentuado \\
\hline
\end{tabular}

5. Exames complementares: os exames mais esclarecedores são os radiológicos que estão descritos no capítulo específico a cada patologia pulmonar (Quadro 4) ${ }^{2}$.

Quadro 4. Diagnóstico diferencial baseado nos achados radiológicos (Adaptado de Bhutani,VK., 1996)²

\begin{tabular}{ll}
\hline Achados & Diagnósticos mais prováveis \\
\hline Aspecto de vidro moído & DMH, S. Pulmão úmido, Pneumonia por Strepto B, Edema pulmonar \\
Bolhas & Enfisema intersticial, DBP, Pneumonia por Stafilococos, Cistos pulmonares \\
Opacificação bilateral & DMH, Hipoplasia pulmonar, Hemorragia pulmonar, derrame pleural \\
Congestão vascular + acúmulo de líquido na cissura & TTRN, S. Pulmão úmido, Hiperfluxo pulmonar \\
Infiltrado grosseiro + atelectasia + hiperinsuflação & Aspiração meconial ou outros líquidos (sangue, leite) \\
Área de hiperinsuflação localizada & Enfisema lobar \\
Ar fora do pulmão & Pneumotórax, Pneumomediastino, Pneumopericárdio \\
Hiperinsuflação pulmonar & Obstrução traqueal parcial, Massa mediastinal \\
\hline
\end{tabular}

\section{CONSIDERAÇÕES FINAIS}

Parece clara, do exposto até aqui, a dificuldade que pode existir no diagnóstico diferencial do desconforto respiratório neonatal, inclusive entre as diversas patologias de origem pulmonar.

É preciso, neste contexto, lembrar da possibilidade de estar-se muitas vezes em presença de um processo patológico de origem multifatorial, o que é, porém, difícil de demonstrar. Na verdade, um recém-nascido que tem os fatores de risco para síndrome de pulmão úmido pode também possuir os de doença de membranas hialinas, como uma produção insuficiente de surfactante. É provável que a concomitância de fatores leve a quadros clínicos em que o diagnóstico preciso necessariamente torna-se problemático.

Esta dificuldade não deverá, porém, prejudicar o cuidado, que será ministrado segundo as necessidades de oxigenação e ventilação, sendo o diagnóstico final uma preocupação muito importante, mas não limitadora da atuação do médico.

Sadeck LSR. Acute respiratory disorders in the neonatal period. Rev Med (São Paulo) 2003 jan.-dez.;82(1-4):40-5.

\begin{abstract}
Neonatal respiratory disorders are frequently present in Neonatal Intensive Care Units, also they are an important cause of death. The acute respiratory disorders can evaluate to chronic disorders (bronchopulmonary dysplasia and chronic lung disease). This review emphasizes the differential dignoses, including epidemiological data, clinical presentation, and radigrafic aspects.
\end{abstract}

KEYWORDS: Respiratory insufficiency/diagnosis. Respiratory insufficiency/complications. Respiratory insufficiency/etiology. Respiratory diseases/diagnosis. Diagnosis, differential. Inafnt, newborn. 


\section{REFERÊNCIAS}

1. Diniz EMA, Sadeck LSR. Diagnóstico diferencial dos distúrbios respiratórios no período neonatal. In: Marcondes E, editor. Pediatria básica. São Paulo: Sarvier; 1991. p.355-9.

2. Bhutani VK. Differential diagnosis of neonatal respiratory disorders. In: Spitzer AR, editor. Intensive care of the fetus and neonate. St Louis: Mosby; 1996. p.494-505.

3. Whitsett JA, Pryhuber GS, Rice WR, Warner BB, Wert SE. Acute respiratory disorders. In: Avery GB, Fletcher MA, MacDonald MG, editors. Neonatology: pathophysiology and management of the newborn. 4th ed. Philadelphia: Lippincott; 1994. p.429-52.

4. Hjalmarson O. Epidemiology and classification of acute, neonatal respiratory disorders. A prospective study. Acta
Paediatr Scand 1981;70:773-83.

5. Stahlman NT. Acute respiratory disorders in the newborn. In: Avery GB, editor. Neonatology: pathophysiology and management of the newborn. 3rd ed. Philadelphia: Lippincott; 1987. p.418-45.

6. Sadeck LSR, Calil VML, Ramos JLA, Leone CR. Insuficiência respiratória aguda no período neonatal. J Pediatria 1990;66(6/7):121-6.

7. Martin RJ, Fanaroff AA. The respiratory distress syndrome and its management. In: Fanaroff AA, Martin RJ, editors. Neonatology. St Louis: Mosby Year Book; 1992. p.81020.

8. Halliday HL, McClure G, McCreid R. Transient tachypnoea of the newborn: two distinct clinical entities? Arch Dis Child 1981;56:322-5. 